\title{
What should we advise about alcohol consumption
}

\author{
Maurizio Ponz de Leon
}

Received: 18 September 2010/Accepted: 30 October 2010/Published online: 30 November 2010 (C) SIMI 2010

I read with interest, and with some concerns, the paper by Castelnuovo et al. [1], and the related commentary [2]. The main message of the review is to present evidence for the protective effect of moderate alcohol intake against cardiovascular diseases. As a consequence, "low-moderate alcohol consumption may contribute to better health".

I do not want to dispute the scientific background of this contention, which could also be well-grounded, though I remain unconvinced of such evidence. What I would like to argue is that the message seems to me hazardous and extremely dangerous to diffuse in the general population, at least for the following reasons:

1. Many people may be unable to distinguish between low-moderate and high consumption of wine, beer or spirits. Moreover, alcohol metabolism may differ remarkably from one subject to another. In current clinical practice, we observe, severe liver or gastric damage associated with relatively low volumes of alcohol (especially in women), whereas other subjects may tolerate without any apparent explanation large amounts of alcohol;

2. Alcohol remains a frequent cause of car crash, and Governments (in almost all Western Countries) try to convince or force people to abstain from drinking before driving. The message of the present review does not help these efforts, since it offers a "moral" justification for drinking, even if in small-moderate amounts;

M. Ponz de Leon ( $\square)$

Dipartimento di Medicine e Specialità Mediche-Medicina I,

Università di Modena e Reggio Emilia, Policlinico,

Via del Pozzo n.71, 41100 Modena, Italy

e-mail: deleon@unimore.it
3. To consider alcohol as a medication whose consumption may contribute to improved health is another source of concern; for instance, the authors state "people who are already regular light-moderate alcohol consumers should be encouraged to continue", but, by the same token, we should encourage the abstemious to drink low-moderate amounts of alcohol, in order to lower HDL, to inhibit platelet aggregation and so on. Are we truly at the point of prescribing alcohol consumption in order to reduce the risk of stroke and coronary damage?

In conclusion, I do believe that more studies are needed before we can give sensible recommendations on alcohol consumption to the general population. Prospective, longterm, cohort investigations are particularly needed. Moreover, even when further evidence of a beneficial effort may be obtained, I would suggest that we use prudence, and even more prudence in dispensing indications for common drinks such as wine, beer or spirits, which are part of our culture, usually make for some joy in our lives, but can also contribute to causing much disease and suffering.

Conflicts of interest None.

\section{References}

1. Di Castelnuovo A, Costanzo S, Donati MB, Iacoviello L, de Gaetano G (2010) Prevention of cardiovascular risk for moderate alcohol consumption: epidemiologic evidence and plausible mechanisms. Intern Emerg Med 5:291-297

2. Estruch R, Lamuela-Raventos RM (2010) Alcohol, wine and cardiovascular disease, two sides of the same coin. Intern Emerg Med 5:277-279 